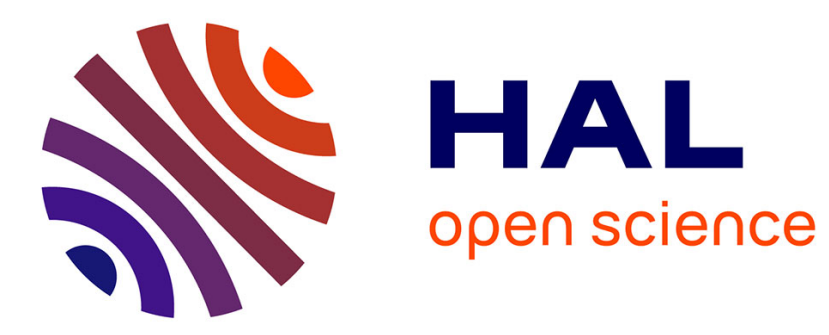

\title{
Observateurs itératifs en horizon fini. Application à la reconstruction de données initiales pour des EDP d'évolution
}

Ghislain Haine, Karim Ramdani

\section{To cite this version:}

Ghislain Haine, Karim Ramdani. Observateurs itératifs en horizon fini. Application à la reconstruction de données initiales pour des EDP d'évolution. Journal Européen des Systèmes Automatisés (JESA), 2011, 45 (7-10), pp.715-724. 10.3166/jesa.45.715-724 . hal-00730518

\section{HAL Id: hal-00730518 \\ https://hal.science/hal-00730518}

Submitted on 14 Feb 2017

HAL is a multi-disciplinary open access archive for the deposit and dissemination of scientific research documents, whether they are published or not. The documents may come from teaching and research institutions in France or abroad, or from public or private research centers.
L'archive ouverte pluridisciplinaire $\mathbf{H A L}$, est destinée au dépôt et à la diffusion de documents scientifiques de niveau recherche, publiés ou non, émanant des établissements d'enseignement et de recherche français ou étrangers, des laboratoires publics ou privés. 


\title{
Observateurs itératifs en horizon fini. Application à la reconstruction de données initiales pour des EDP d'évolution.
}

\author{
Ghislain Haine* - Karim Ramdani** \\ * Université Henri Poincaré (Institut Élie Cartan) \\ B.P. 70239, 54506 Vandoeuvre-les-Nancy, France \\ Ghislain.Haine@univ-lorraine.fr \\ ** INRIA Nancy Grand-Est (CORIDA) \\ 615 rue du Jardin Botanique, 54600 Villers-les-Nancy, France \\ Karim.Ramdani@inria.fr
}

\begin{abstract}
RÉSUMÉ. Un nouvel algorithme itératif pour la reconstruction de la donnée initiale d'un système dynamique à partir de mesures partielles a récemment été proposé dans l'article de Ramdani, Tucsnak et Weiss (Ramdani et al., 2010). Cet algorithme, qui permet de traiter des systèmes linéaires de dimensions finie ou infinie, consiste à utiliser de manière itérative deux observateurs de Luenberger en horizon fini, l'un direct et l'autre rétrograde. Dans cet article, nous expliquons le principe de cet algorithme en dimension finie avant de le généraliser aux Équations aux Dérivées Partielles (EDP). Enfin, nous le mettons en oeuvre numériquement sur l'exemple de l'équation de la corde vibrante.

ABSTRACT. A new iterative algorithm for solving initial data inverse problems from partial observations has been recently proposed in Ramdani, Tucsnak and Weiss (Ramdani et al., 2010). The algorithm, which can be used for finite-dimensional and infinite-dimensional linear systems, is based on the use of two observers (also called Luenberger observers) used iteratively back and forth in time. In this paper, we first present the algorithm in a finite-dimensional context and then generalize it to Partial Differential Equations (PDE). To conclude, the method is applied to the $1 D$ wave equation and numerical results will be given.
\end{abstract}

MOTS-CLÉS : Observateurs, EDP d'évolution, équation des ondes.

KEYWORDS: Observers, Evolution PDE, wave equation.

JESA - 45/2011. Contrôle des systèmes à paramètres répartis, pages 715 à 724 


\section{Introduction}

Soient $n \in \mathbb{N}^{*}$, et $A \in M_{n}(\mathbb{C})$. On considère le système dynamique

$$
\dot{z}(t)=A z(t), \quad \forall t \geq 0,
$$

où $z(t)$ désigne l'état du système et $\dot{z}(t)$ sa dérivée temporelle à l'instant $t$. On suppose que l'on dispose d'une mesure partielle $y(t)$ obtenue à partir de l'état du système via un opérateur linéaire d'observation $C \in M_{n, m}(\mathbb{C}), m \in \mathbb{N}^{*}$ (éventuellement distinct de $n)$ :

$$
y(t)=C z(t), \quad \forall t \geq 0 .
$$

Le problème auquel on s'intéresse ici est le problème inverse de la reconstruction de l'état initial du système $z_{0}=z(0)$ à partir de la connaissance de la sortie $y(t)$. En d'autres termes, il s'agit de résoudre le système linéaire (rectangulaire) $C e^{t A} z_{0}=y(t)$. La résolution de cette équation par inversion du grammien d'observabilité du système est coûteuse et conduit à la résolution de systèmes linéaires mal conditionnés. En temps long (horizon infini), les observateurs de Luenberger (Luenberger, 1964) constituent une solution alternative intéressante. En effet, à l'aide d'un observateur, on peut dans un premier temps reconstruire l'état du système, sous réserve que celui-ci soit détectable ${ }^{1}$. À partir de cette estimation, il est possible de reconstruire l'état initial via un observateur de type rétrograde (i.e. un observateur usuel du système après retournement temporel) à condition que le système rétrograde $(-A, C)$ soit également détectable. Dans cet article, on suppose que la sortie $y(t)$ est connue uniquement sur un intervalle de temps fini $[0, \tau]$ (horizon fini), de sorte que la méthode précédemment décrite n'est plus opérante. Nous en proposons une adaptation naturelle consistant à utiliser de manière itérative les deux observateurs précédemment décrits (voir la figure 1 pour une représentation schématique de l'algorithme itératif et l'équation [8] pour une description précise). Soulignons que cet algorithme a déjà été proposé par Auroux et Blum (Auroux et al., 2008) dans le contexte des problèmes d'assimilation de données. Toutefois, l'étude de la convergence n'a été menée que dans le cadre d'une observation totale dans (Auroux et al., 2005).

L'article est organisé de la façon suivante : nous présentons dans la section 2 l'algorithme en dimension finie. La section 3 donne les principaux ingrédients nécessaires au passage en dimension infinie, c'est-à-dire au cadre des EDP. Enfin, nous concluons par l'étude de l'équation de la corde vibrante dans la section 4. En particulier, nous verrons qu'elle relève du cadre abstrait décrit en amont (section 4.1) et nous présenterons des résultats numériques de reconstruction (section 4.2).

1. $(A, C)$ est dit détectable si et seulement s'il existe une matrice de gain $H \in M_{m, n}(\mathbb{C})$ telles que $A+H C$ soit de Hurwitz (i.e. son spectre $\sigma(A)$ est situé dans le demi-plan gauche ouvert de $\mathbb{C})$. En particulier, tout système observable est détectable. 


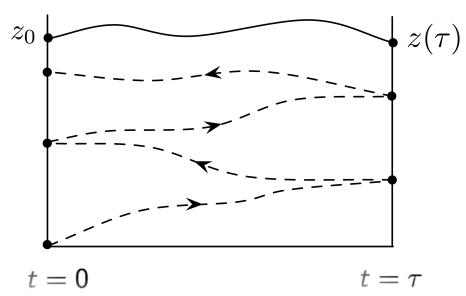

Figure 1. Observateurs itératifs

\section{Systèmes en dimension finie}

On suppose que le système $(A, C)$ est détectable dans les sens direct et rétrograde. Autrement dit, on suppose qu'il existe deux matrices de gain $H^{+}, H^{-} \in M_{m, n}(\mathbb{C})$ telles que $A^{+}=A+H^{+} C$ et $A^{-}=-A+H^{-} C$ soient de Hurwitz. Nous allons voir que sous cette hypothèse (et en s'assurant que [6] est vérifiée), on peut obtenir un algorithme itératif de reconstruction de $z_{0}$.

Si l'on note $\omega^{+}=\max _{\lambda \in \sigma\left(A^{+}\right)} \operatorname{Re} \lambda<0$ et $\omega^{-}=\max _{\lambda \in \sigma\left(A^{-}\right)} \operatorname{Re} \lambda<0$, et que l'on choisit $\omega$ de sorte que $\max \left\{\omega^{+}, \omega^{-}\right\}<\omega<0$, alors il existe un $M_{\omega}>0$ tel que

$$
\begin{cases}\left\|e^{t A^{+}} z\right\| \leq M_{\omega} e^{\omega t}\|z\|, & \forall z \in C, \\ \left\|e^{t A^{-}} z\right\| \leq M_{\omega} e^{\omega t}\|z\|, & \forall z \in C .\end{cases}
$$

Considérons alors l'observateur (direct)

$$
\left\{\begin{array}{l}
\dot{z}^{+}(t)=A^{+} z^{+}(t)-H^{+} y(t), \quad \forall t \in[0, \tau], \\
z^{+}(0)=z_{0}^{+} \in \mathbb{C}^{n},
\end{array}\right.
$$

ainsi que l'observateur rétrograde

$$
\left\{\begin{array}{l}
\dot{z}^{-}(t)=-A^{-} z^{-}(t)+H^{-} y(t), \quad \forall t \in[0, \tau], \\
z^{-}(\tau)=z^{+}(\tau) \in \mathbb{C}^{n},
\end{array}\right.
$$

où $\tau>0$ est suffisamment grand pour que

$$
\alpha_{\omega}:=M_{\omega} e^{\omega \tau}<1
$$

Posons $e^{+}(t)=z^{+}(t)-z(t)$, on a alors

$$
\begin{aligned}
\dot{e}^{+}(t) & =\dot{z}^{+}(t)-\dot{z}(t), \\
& =A^{+} z^{+}(t)-H^{+} C z(t)-A z(t), \\
& =A^{+} z^{+}(t)-A^{+} z(t)=A^{+} e^{+}(t),
\end{aligned}
$$

d'où $e^{+}(\tau)=e^{\tau A^{+}} e^{+}(0)$. Posant $e^{-}(t)=z^{-}(t)-z(t)$, on a également $\dot{e}^{-}(t)=$ $-A^{-} e^{-}(t)$, ce qui nous donne $e^{-}(0)=e^{\tau A^{-}} e^{-}(\tau)$. Or $e^{+}(\tau)=e^{-}(\tau)$, donc

$$
e^{-}(0)=e^{\tau A^{-}} e^{\tau A^{+}} e^{+}(0),
$$


ou encore

$$
z^{-}(0)-z_{0}=e^{\tau A^{-}} e^{\tau A^{+}}\left(z_{0}^{+}-z_{0}\right)
$$

ce qui implique par [3] que

$$
\left\|z^{-}(0)-z_{0}\right\| \leq \alpha_{\omega}^{2}\left\|z_{0}^{+}-z_{0}\right\|
$$

Comme cela a déjà été dit, on voit qu'en horizon infini, $z^{-}(0)$ constitue une bonne approximation de l'état initial recherché. Si on se place en horizon fini, il suffit d'itérer le processus décrit ci-dessus. Plus précisément, en résolvant de nouveau [4] et [5] mais en posant cette fois-ci $z_{0}^{+}=z_{1}^{-}(0)$ (la première estimation de $z_{0}$ ), on obtient une nouvelle estimée de $z_{0}$ notée $z_{2}^{-}(0)$. Par [7], on obtient

$$
\left\|z_{2}^{-}(0)-z_{0}\right\| \leq \alpha_{\omega}^{2}\left\|z_{1}^{-}(0)-z_{0}\right\| \leq \alpha_{\omega}^{4}\left\|z_{0}^{+}-z_{0}\right\| .
$$

De cette façon, en itérant $N$ fois ce procédé, on obtient par récurrence l'estimation suivante pour la qualité de la reconstruction

$$
\left\|z_{N}^{-}(0)-z_{0}\right\| \leq \alpha_{\omega}^{2 N}\left\|z_{0}^{+}-z_{0}\right\|
$$

de sorte que, grâce à [6], la différence entre la donnée reconstruite $z_{N}^{-}(0)$ et la donnée initiale $z_{0}$ tend bien vers zéro exponentiellement quand le nombre d'itérations $N$ tend vers l'infini.

En résumé, l'algorithme de reconstruction consiste à résoudre de manière itérative deux équations d'évolution (à savoir [4] et [5]) ayant un terme de rappel à la mesure et dont seules les conditions initiales ou finales changent. Plus précisément, l'algorithme itératif prend la forme suivante

$$
\begin{gathered}
\left\{\begin{array}{lr}
\dot{z}_{n}^{+}(t)=A^{+} z_{n}^{+}(t)-H^{+} y(t), & \forall t \in[0, \tau] \\
z_{n}^{+}(0)=z_{n-1}^{-}(0) \text { pour } n \geq 1, & z_{0}^{+}(0)=z_{0}^{+}
\end{array}\right. \\
\left\{\begin{array}{l}
\dot{z}_{n}^{-}(t)=-A^{-} z_{n}^{-}(t)+H^{-} y(t), \\
z_{n}^{-}(\tau)=z_{n}^{+}(\tau)
\end{array}\right.
\end{gathered}
$$

\section{Généralisation à la dimension infinie}

Les définitions d'analyse fonctionnelle utilisées dans cette section pourront être lues dans Tucsnak et Weiss (Tucsnak et al., 2009).

On se restreint dans cette section au cadre fonctionnel correspondant à l'exemple numérique traité dans la section 4. Plus précisément, soient $X$ un espace de Hilbert (dit espace des états), $A: \mathcal{D}(A) \rightarrow X$ un opérateur anti-adjoint (la contre partie infini-dimensionnelle des matrices hilbertiennes) et $C \in \mathcal{L}(X, Y)$ un opérateur d'observation, où $Y$ est un autre espace de Hilbert (dit espace des sorties). On considère alors

$$
\left\{\begin{array}{l}
\dot{z}(t)=A z(t), \quad \forall t \geq 0, \\
z(0)=z_{0} \in \mathcal{D}(A)
\end{array}\right.
$$




$$
y(t)=C z(t), \quad \forall t \geq 0 .
$$

Pour pouvoir adapter la méthode décrite dans la section 2, il faut généraliser le concept de détectabilité. Autrement dit, il faut trouver des opérateurs $H^{+}$et $H^{-}$de telle sorte que $A^{+}=A+H^{+} C$ et $A^{-}=-A-H^{-} C$ conduisent à des systèmes exponentiellement stables. Soulignons que contrairement au cas de la dimension finie, le fait que le spectre d'un opérateur soit dans le demi-plan gauche ne suffit plus à obtenir la stabilité exponentielle du système associé.

En dimension finie, l'existence de $\mathrm{H}^{+}$et $\mathrm{H}^{-}$est par exemple assurée par le caractère observable de $(A, C)$ (voir (Trélat, 2005, p.232) ou (Sontag, 1998, p. 316)). Pour transposer ce résultat à la dimension infinie, on introduit la notion d'observabilité exacte d'un système. On dira que $(A, C)$ est exactement observable en temps $\tau$ si et seulement s'il existe une constante $\delta>0$ telle que

$$
\int_{0}^{\tau}\|y(t)\|^{2} d t \geq \delta\left\|z_{0}\right\|^{2} \quad \forall z_{0} \in \mathcal{D}(A) .
$$

REMARQUe. - On notera qu'en dimension infinie, l'observabilité exacte en temps $\tau>0$ ne l'implique pas en général en temps $t<\tau$. L'exemple le plus courant étant celui de l'équation des ondes sur un domaine borné, où le temps minimal d'observation nécessaire pour l'observabilité exacte est égal au temps nécessaire pour que tout rayon dans le domaine atteigne la région d'observation. Cette condition est appelée condition d'optique géométrique de Bardos, Lebeau et Rauch (Bardos et al., 1992). Par exemple, si le domaine d'observation est un voisinage de tout le bord du domaine, le temps minimal sera inférieur à $D / c$ où $D$ est le plus grand diamètre du domaine et $c$ la vitesse de propagation des ondes dans le milieu. Il faudra donc veiller dans le cadre des EDP à mesurer sur un intervalle de temps suffisamment long (ou à prendre une zone d'observation suffisamment grande) pour garantir l'observabilité exacte.

Dans le cadre infini-dimensionnel décrit ci-dessus, l'observabilité exacte en temps $\tau$ implique encore la convergence de l'algorithme itératif. En effet, en supposant que $(A, C)$ est exactement observable en $\tau>0$, le théorème 2.3. de Liu (Liu, 1997) nous dit que l'on peut choisir $H^{+}=H^{-}=-\gamma C^{*}$, pour tout $\gamma>0$ (coefficient de gain), pour obtenir la stabilité exponentielle des systèmes associés à $A^{+}=A-\gamma C^{*} C$ et $A^{-}=-A-\gamma C^{*} C$. Passée cette difficulté sur l'existence de $H^{+}$et $H^{-}$, la suite de la méthode itérative est identique au cas de la dimension finie (moyennant quelques détails techniques, la notion d'exponentielle de matrice étant par exemple remplacée par celle de semi-groupe).

Dans le cas plus général où $A: \mathcal{D}(A) \rightarrow X$ n'est pas anti-adjoint mais seulement le générateur infinitésimal d'un $C_{0}$-semigroupe et où $C$ n'est pas borné (i.e. $C \in \mathcal{L}(\mathcal{D}(A), Y)$ ), il n'existe pas d'équivalent du théorème de Liu permettant de trouver $H^{+}$et $H^{-}$sous l'hypothèse que $(A, C)$ est exactement observable en temps $\tau>0$. L'existence de ces opérateurs devient alors une hypothèse, dite hypothèse 
d'estimabilité directe et rétrograde, remplaçant ainsi celle d'observabilité exacte. La définition exacte de ce concept est donnée dans (Ramdani et al., 2010).

\section{Un exemple : l'équation de la corde vibrante}

\subsection{Le problème continu}

Dans cette section, nous considérons l'équation de la corde vibrante sur l'intervalle $I=(0,1)$, avec condition aux bords de Dirichlet (les extrémités de la corde sont fixées) et dont tous les coefficients sont normalisés à 1 (en particulier la vitesse de propagation de l'onde). Autrement dit

$$
\begin{cases}\frac{\partial^{2} w}{\partial t^{2}}(x, t)=\frac{\partial^{2} w}{\partial x^{2}}(x, t), & \forall t \geq 0, \forall x \in I, \\ w(0, t)=w(1, t)=0, & \forall t \geq 0, \\ w(x, 0)=w_{0}(x), \frac{\partial w}{\partial t}(x, 0)=w_{1}(x), & \forall x \in I .\end{cases}
$$

où $w_{0}(x)$ et $w_{1}(x)$ sont respectivement la position et la vitesse initiale de la corde. On suppose que l'on dispose de la mesure de la dérivée au voisinage de 0 (c'est-à-dire sur un intervalle $[0, \alpha], 0<\alpha<1$ ) sur un intervalle de temps [0, $\tau]$ (la valeur du paramètre $\tau$ sera discutée plus loin), c'est-à-dire

$$
y(x, t)=\frac{\partial w}{\partial t}(x, t), \quad \forall(x, t) \in[0, \alpha] \times[0, \tau] .
$$

Le problème étudié est le suivant : peut-on reconstruire le couple $\left(w_{0}(x), w_{1}(x)\right)$ à partir de $y(t)$ ?

Dans un premier temps, il faut donner le bon cadre fonctionnel permettant d'obtenir l'existence et l'unicité d'une solution au système [11]. Rappelons pour cela la définition de quelques espaces fonctionnels classiques :

- $L^{2}(I)$ l'espace des fonctions de carré intégrable sur $I$

- L'espace de Sobolev $H^{1}(I)=\left\{f \in L^{2}(I) \mid \frac{d f}{d x} \in L^{2}(I)\right\}$

- L'espace de Sobolev $H_{0}^{1}(I)=\left\{f \in H^{1}(I) \mid f(0)=f(1)=0\right\}$

- L'espace de Sobolev $H^{2}(I)=\left\{f \in H^{1}(I) \mid \frac{d^{2} f}{d x^{2}} \in L^{2}(I)\right\}$

Alors si $\left(w_{0}(x), w_{1}(x)\right) \in\left(H^{2}(I) \cap H_{0}^{1}(I)\right) \times H_{0}^{1}(I)$, il existe une unique solution $w(x, t)$ vérifiant (Tucsnak et al., 2009, théorème 4.1.6.)

$$
w \in C\left(\left[0, \infty\left[, H^{2}(I)\right) \cap C^{1}\left(\left[0, \infty\left[, H_{0}^{1}(I)\right) \cap C^{2}\left(\left[0, \infty\left[, L^{2}(I)\right) .\right.\right.\right.\right.\right.\right.
$$

Nous allons maintenant réécrire [11]-[12] sous la forme générale [9]-[10]. Posons $H=L^{2}(I), A_{0} f=-\frac{d^{2} f}{d x^{2}}$ défini sur $H_{1}=\mathcal{D}\left(A_{0}\right)=H^{2}(I) \cap H_{0}^{1}(I)$ à valeurs dans 
$H, H_{\frac{1}{2}}=H_{0}^{1}(I)$ et $C_{0} f=\chi f$, où $\chi$ est une fonction plateau de classe $\mathcal{C}^{\infty}$ valant 1 sur $[0, \alpha]$ et 0 sur le complémentaire d'un voisinage de $[0, \alpha]$. Alors en notant

$$
\begin{gathered}
z(t)=\left[\begin{array}{c}
w(t) \\
\dot{w}(t)
\end{array}\right], \quad X=H_{\frac{1}{2}} \times H, \\
A=\left(\begin{array}{cc}
0 & I \\
-A_{0} & 0
\end{array}\right), \quad \mathcal{D}(A)=H_{1} \times H_{\frac{1}{2}}, \\
C=\left[\begin{array}{ll}
0 & C_{0}
\end{array}\right], \quad Y=H,
\end{gathered}
$$

le système [11]-[12] se réécrit sous la forme [9]-[10].

Pour pouvoir appliquer l'algorithme de reconstruction, il faut encore trouver les deux opérateurs $\mathrm{H}^{+}$et $\mathrm{H}^{-}$permettant de considérer les observateurs direct et rétrograde [4] et [5] dans le cadre des équations aux dérivées partielles. Or dans le cas que l'on considère ici (voir par exemple (Tucsnak et al., 2009)), l'opérateur $A$ est antiadjoint et $C \in \mathcal{L}(X, Y)$. Le théorème de Liu (Liu, 1997) s'applique donc si l'on peut montrer qu'il existe un $\tau>0$ tel que $(A, C)$ soit exactement observable en $\tau$. D'après la condition d'optique géométrique, il faut que tout rayon de l'intervalle $I$ atteigne la région d'observation $[0, \alpha]$. Dans notre cas, un rayon du domaine ne peut suivre que deux directions : soit vers 0 , soit vers 1 . Si le rayon se dirige vers 0 , il rencontrera donc notre région d'observation, et s'il va vers 1 , les principes de l'optique géométrique le réfléchiront vers 0 . Ainsi, avec une vitesse de propagation constante égale à $1, \tau=2$ est suffisant pour que tout rayon ait rencontré la région $[0, \alpha]$. En d'autres termes, $(A, C)$ est exactement observable en $\tau=2$. Alors le théorème de Liu s'applique et l'on peut prendre $H^{+}=H^{-}=-\gamma C^{*}$ pour utiliser notre algorithme et reconstruire la donnée initiale $\left(w_{0}, w_{1}\right)$.

\subsection{Discrétisation et tests numériques}

Nous allons maintenant présenter quelques résultats numériques sur l'exemple de l'équation de la corde vibrante de la section 4.1 obtenus par la discrétisation des observateurs continus [8]. Prenons $\alpha=0.1, \tau=2, w_{0}(x)=w_{1}(x)=2 \sin (4 \pi x)$ et $H^{+}=H^{-}=-\gamma \chi$, où $\gamma>0$ est le gain. Nous discrétisons les observateurs direct et rétrograde [8] en utilisant des éléments finis en espace (paramètre de discrétisation $h$ ), et par différence finie implicite en temps (paramètre de discrétisation $\Delta t$ ). Pour plus de détails sur les schémas utilisés et l'analyse détaillée de leur convergence, nous renvoyons le lecteur à Haine et Ramdani (Haine et al., 2012). Notons simplement qu'il existe un nombre d'itérations $N_{h, \Delta t}$ optimal dépendant des pas de discrétisation, permettant d'obtenir une erreur en $O\left(\left(h^{2}+\Delta t\right) \ln ^{2}\left(h^{2}+\Delta t\right)\right)$.

REMARQUE. - Pour générer la mesure $y(t)$ utilisée dans les simulations, nous choisissons une donnée initiale nous permettant de calculer analytiquement la solution exacte du système [11] à tout instant. Cette précaution est prise afin d'éviter le risque de commettre un crime inverse ${ }^{2}$.

2. On désigne par ce terme des reconstructions dont l'excellente qualité est un artefact dû à une interaction entre les schémas numériques utilisés pour résoudre les problèmes direct (pour générer les données) et inverse ; voir par exemple Colton et Kress (Colton et al., 1998). 

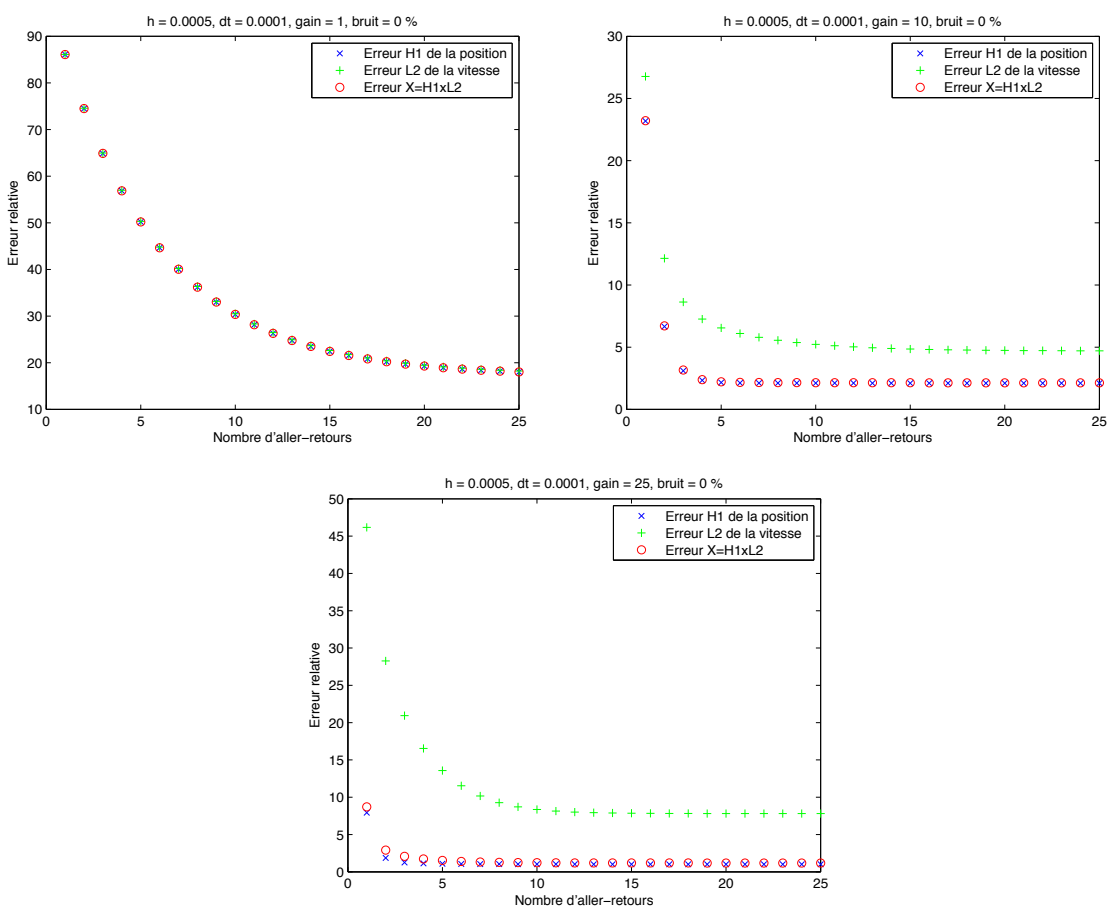

Figure 2. Influence du gain : $\gamma=1,10,25$

Les simulations numériques effectuées montrent qu'en faisant varier le paramètre de gain $\gamma$, on peut sensiblement agir sur le taux de décroissance exponentielle de l'erreur (voir figure 2). Ainsi dès la première itération, l'erreur relative globale (dans $X$ ) est de l'ordre de $10 \%$ pour $\gamma=25$, de $23 \%$ pour $\gamma=10$ alors qu'elle est proche de $90 \%$ pour $\gamma=1$. Nous choisirons dorénavant un gain $\gamma=10$, qui est proche de la valeur optimale permettant de réduire le plus rapidement et simultanément les erreurs relatives sur la position et la vitesse. On voit sur la figure 3 qu'on améliore bien la qualité de la reconstruction en discrétisant plus finement. La figure 4 permet d'apprécier la qualité de la reconstruction des données initiales inconnues à la convergence (après 25 itérations pour $h=0,0002$ et $d t=0,00004$ ). Enfin, l'algorithme semble assez robuste vis-à-vis du bruit, comme le montre la figure 5 représentant l'erreur relative pour différentes valeurs du bruit (respectivement de $5 \%$ et de $20 \%$ environ). 

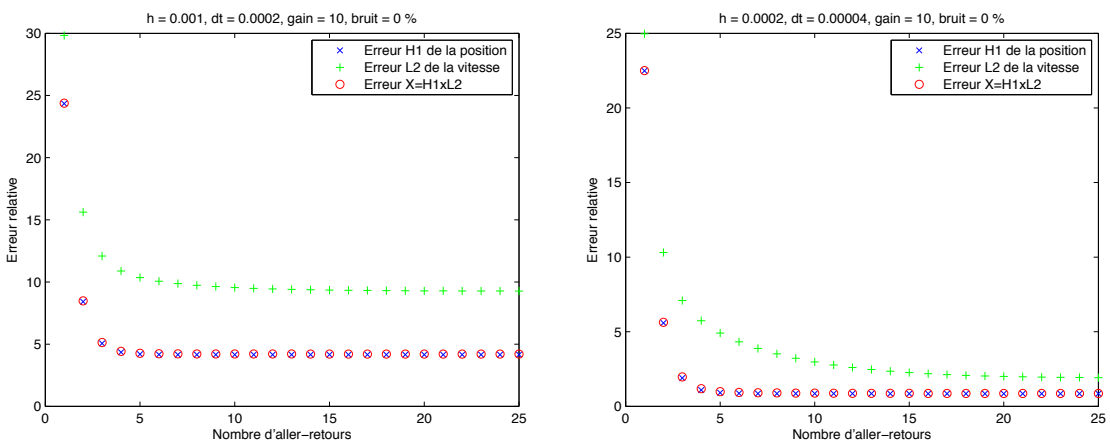

Figure 3. Influence des pas de discrétisation sur l'erreur optimale
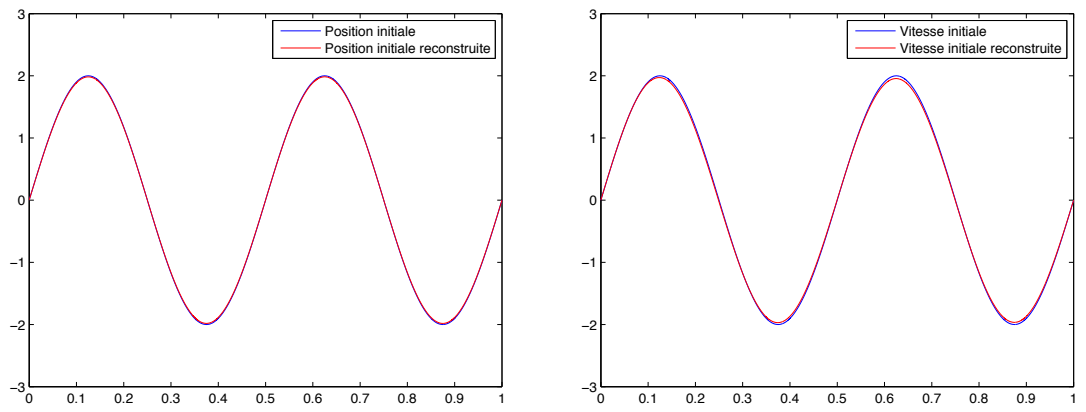

Figure 4. Reconstruction de la position (à gauche) et de la vitesse (à droite)
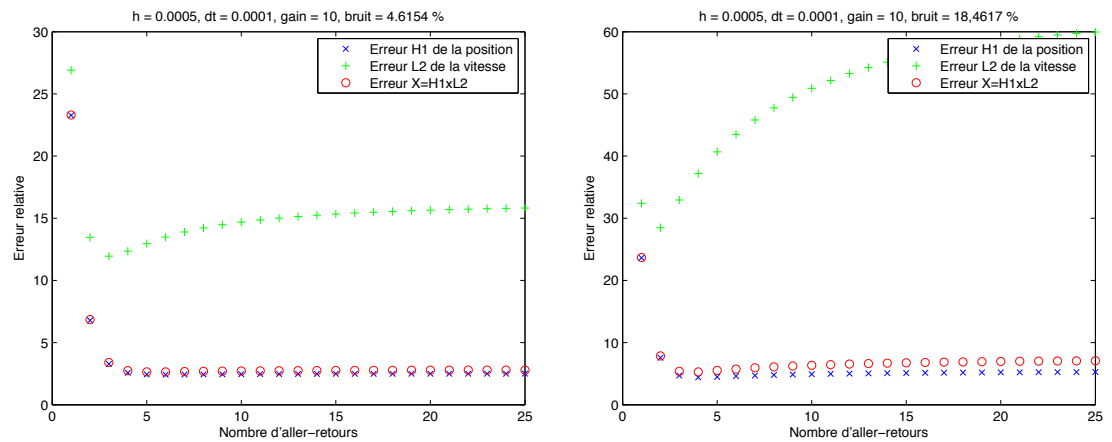

Figure 5. Influence du bruit : bruit relatif de $5 \%$ (à gauche) et de $20 \%$ (à droite) 


\section{Conclusion}

Nous avons présenté une méthode originale pour reconstruire la donnée initiale d'un système d'évolution linéaire de dimension finie ou infinie, à partir de mesures partielles et sur un intervalle de temps fini. L'idée principale de notre approche consiste à itérer des observateurs direct et rétrograde, conduisant à une approximation exponentiellement précise de la donnée initiale. Enfin, nous avons présenté des résultats numériques illustrant l'efficacité de notre algorithme itératif. L'analyse de convergence de cet algorithme a été menée dans (Haine et al., 2012) pour une observation interne (opérateur d'observation borné), le cas des observations frontière (opérateur d'observation non borné) restant ouvert. Une question naturelle consisterait à étudier la généralisation des résultats présentés ici au cas des systèmes semi-linéaires ou non linéaires.

\section{Bibliographie}

Auroux D., Blum J., « Back and forth nudging algorithm for data assimilation problems », C. R. Math. Acad. Sci. Paris, vol. 340, n 12, p. 873-878, 2005.

Auroux D., Blum J., « A nudging-based data assimilation method : the Back and Forth Nudging (BFN) algorithm », Nonlin. Proc. Geophys., 2008.

Bardos C., Lebeau G., Rauch J., « Sharp sufficient conditions for the observation, control, and stabilization of waves from the boundary », SIAM J. Control Optim., vol. 30, n 5, p. 1024$1065,1992$.

Colton D., Kress R., Inverse acoustic and electromagnetic scattering theory, Applied Mathematical Sciences, second edn, Springer-Verlag, Berlin, vol. 93, 1998.

Haine G., Ramdani K., « Reconstructing initial data using observers : error analysis of the semi-discrete and fully discrete approximations », Numerische Mathematik, vol. 120, $\mathrm{n}^{\circ} 2$, p. 307-343, 2012.

Liu K., « Locally distributed control and damping for the conservative systems », SIAM J. Control Optim., vol. 35, n 5, p. 1574-1590, 1997.

Luenberger D., « Observing the state of a linear system », IEEE Trans. Mil. Electron., vol. MIL8, p. 74-80, 1964.

Ramdani K., Tucsnak M., Weiss G., « Recovering the initial state of an infinite-dimensional system using observers », Automatica, vol. 46, n 10, p. 1616-1625, 2010.

Sontag E. D., Mathematical control theory, Texts in Applied Mathematics, second edn, SpringerVerlag, New York, vol. 6, 1998. Deterministic finite-dimensional systems.

Trélat E., Contrôle optimal, Mathématiques Concrètes, Vuibert, Paris, 2005. Théorie \& applications.

Tucsnak M., Weiss G., Observation and control for operator semigroups, Birkhäuser Advanced Texts : Basler Lehrbücher., Birkhäuser Verlag, Basel, 2009. 